\title{
X-ray Microanalysis at High Count Rate with Latest Generation Silicon Drift Energy Dispersive Spectrometer
}

\author{
Philippe T. Pinard ${ }^{1}$, Simon Burgess ${ }^{1}$, John Qing Zhang ${ }^{1}$, James Holland ${ }^{1}$ and Peter Statham ${ }^{1}$ \\ 1. Oxford Instruments NanoAnalysis, High Wycombe, UK.
}

Silicon drift detectors (SSD) undeniably have several advantages over the former Si(Li) technology for energy dispersive spectrometry. On top of this list is their excellent energy resolution at high count rates. Each new generation of SSD has pushed these count rate capabilities [1]. Advancements in electronics have progressively reduced the electronic noise, meaning that shorter processing times can be used to increase the throughput without compromising the spectral resolution. Although important in assessing the electronic performance of a spectrometer, the count rate and spectral resolution are not the only parameters that will influence the quality and accuracy of a measurement. This is especially true at high input count rates where phenomena such as electronic instability, ballistic deficit and pulse pile-up may have detrimental effects on both qualitative and quantitative analyses [2]. In this study we have considered four critical factors essential for accurate X-ray mapping and quantification at input count rates above $350 \mathrm{kHz}$ : (1) detailed characterization of X-ray detector, (2) accurate theoretical description of peak profiles, (3) stable position and resolution of peaks and (4) effective pulse pile-up correction.

The capabilities of modern X-ray mapping software goes beyond creating X-ray images from energy windows. Processing of spectral mapping can differentiate elements from overlapping peaks, remove the influence of the continuum, identify phases and provide quantitative information [2-3]. A key component of this processing is the calculation of peak profiles for each X-ray peak of every element, taking into account the detector characteristics as well as the accelerating voltage and processing parameters used during the acquisition. The filtered least-squares algorithm relies on an accurate theoretical description of the experimental X-ray peaks in order to resolve overlapping peaks and extract accurate peak areas. Therefore, the position, resolution and shape of the X-ray peaks in the spectral mapping data must remain constant for all phases for any sample at any count rate. Figure 1 shows an example of a multi-phase mineral sample. The use of peak profiles is necessary to separate the overlapping $\mathrm{Mg} \mathrm{K}$ and As L series and to properly identify arsenopyrite and biotite mica. The stability of the X-ray peaks is confirmed since the input count rate from arsenopyrite $(640 \mathrm{kHz})$ is $50 \%$ greater than the one of biotite mica $(425 \mathrm{kHz})$.

Besides increasing productivity and decreasing the concentration limit of detection, high count rate acquisition allows moving to a mapping-centric workflow. X-ray maps are acquired instead of individual spectra, and spectra from specific phases or regions are extracted from a map for a detailed quantification. The requirements for accurate quantitative analysis therefore necessarily overlap with those of X-ray mapping. As the count rate increases, the probability of pulse pile-up events increases, resulting in larger and higher order sum peaks. Unless completely corrected, sum peaks may lead to identification of false elements, reduced intensity of major elements and increased intensity of minor elements. One way to verify the effectiveness of spectrum stability, correction and processing, and in particular the pulse pile-up correction is to acquire spectra at different input count rates and compare the quantification results. Table 1 compares the standardless, unnormalized results of three acquisitions at input count rates of 4, 200 and $400 \mathrm{kHz}$ acquired on a homogeneous orthoclase standard at $20 \mathrm{kV}$. Figure 2 shows the raw and corrected spectrum at $400 \mathrm{kHz}$. Orthoclase is a useful test because of the 
pile-up of major elements lines as sum peaks at the energy of other lines, for example Al and Si pile-up creates a complex overlap with another major element $\mathrm{K}$ and a trace constituent $\mathrm{Ca}$. The variability found is less than $1 \%$ for major elements $(\mathrm{O}, \mathrm{Al}, \mathrm{Si})$, less than $3 \%$ for minor elements $(\mathrm{Na}$ and $\mathrm{K})$ and approximately $10 \%$ for the trace element $(\mathrm{Ca})$. This confirms that the sum peaks were properly corrected by putting their counts back in the correct spectrum channels.

\section{References:}

[1] D Newbury and NWM Ritchie, Microsc. Microanal. 22 (2016), p. 520.

[2] S Burgess et al, Microsc. Microanal. 23(S1) (2017), p. 46.

[3] R Wuhrer and K Moran, IOP Conf. Ser.: Mater. Sci. Eng. 55 (2014), 012021.

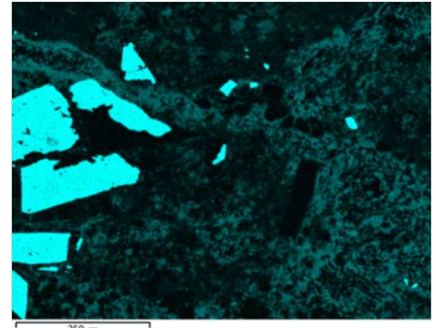

(a) As L

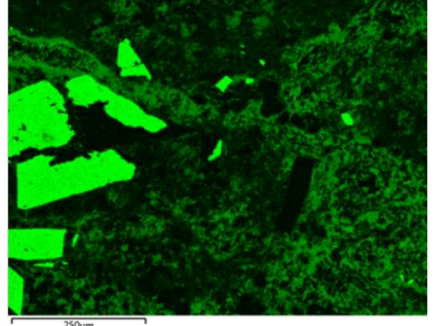

(b) $\mathrm{Mg} \mathrm{K}$

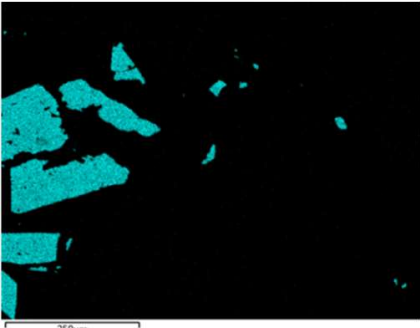

(c) As L

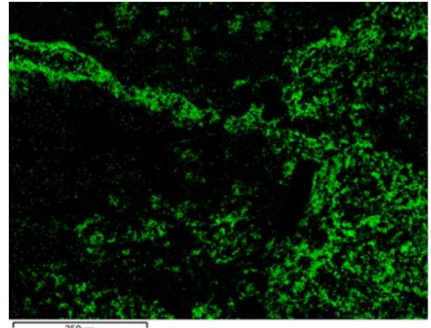

(d) $\mathrm{Mg} \mathrm{K}$

Figure 1. Comparison between energy window integral (a-b) and peak area (c-d) X-ray images from a multiphase sample containing As (a,c) and $\mathrm{Mg}(\mathrm{b}, \mathrm{d})$. While the energy window integral images of As and $\mathrm{Mg}$ look almost identical, the distribution of arsenopyrite and biotite mica phases is clearly visible in the peak area images. The X-ray map was acquired for $500 \mathrm{~s}$ live time at $20 \mathrm{kV}$ with an UltimMax 170 detector on a Tescan Mira3 microscope (PT2, $2048 \mathrm{ch}, 20 \mathrm{keV}$ energy range, takeoff angle of $35^{\circ}$ ).

\begin{tabular}{|c|c|c|c|c|c|c|c|c|}
\hline $\begin{array}{c}\text { Input count } \\
\text { rate (kHz) }\end{array}$ & $\begin{array}{c}\text { Output count } \\
\text { rate (kHz) }\end{array}$ & O & Na & Al & Si & K & Ca & Total \\
\hline $\mathbf{4}$ & $\mathbf{4}$ & 46.08 & 2.73 & 10.33 & 31.18 & 9.44 & 0.22 & 99.98 \\
\hline $\mathbf{2 0 0}$ & $\mathbf{1 4 0}$ & 46.17 & 2.76 & 10.4 & 31.38 & 9.43 & 0.19 & 100.33 \\
\hline $\mathbf{4 0 0}$ & $\mathbf{2 0 0}$ & 46.41 & 2.88 & 10.47 & 31.64 & 9.25 & 0.18 & 100.84 \\
\hline
\end{tabular}

Table 1. Standardless, unnormalized quantification results (in wt.\%) of an orthoclase standard (carbon coated) acquired at $20 \mathrm{kV}$ with the same system as Figure 1 (PT3, 5 million counts). All elements were automatically identified, except for $\mathrm{Ca}$. Co was used for beam calibration.

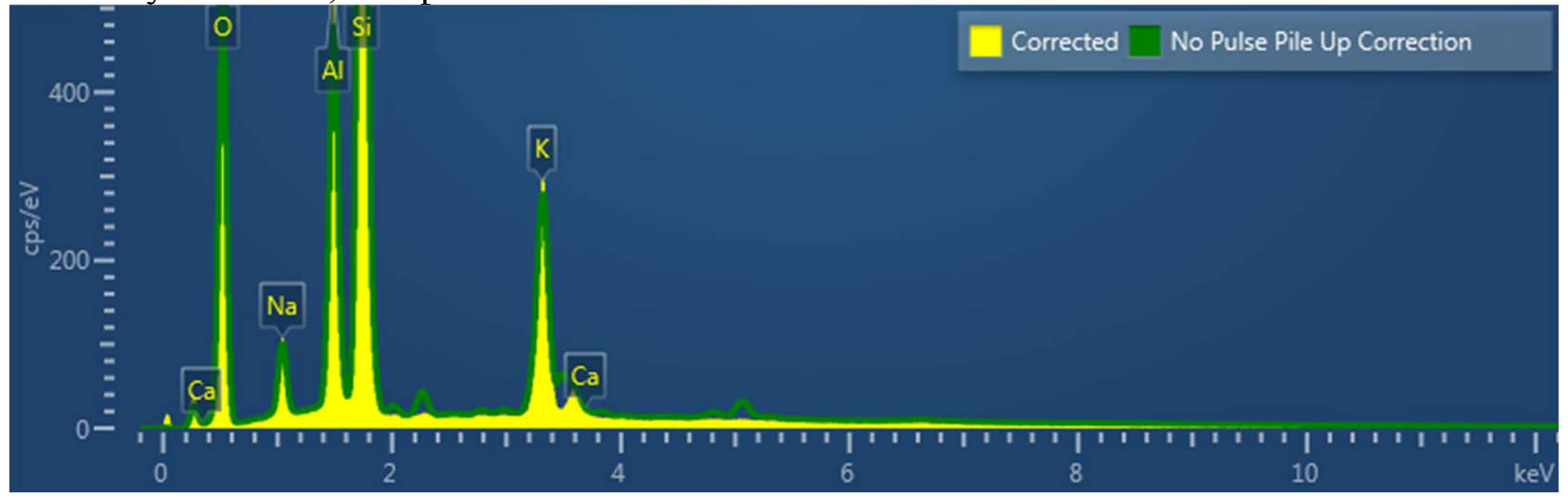

Figure 2. Spectrum from a homogeneous orthoclase standard (carbon coated) at $20 \mathrm{kV}$ with and without pile-up correction acquired at input count rate of $400 \mathrm{kHz}$ and same acquisition conditions as Table 1. 